\title{
Modeling the interplay between Solvent Evaporation and Phase Separation Dynamics during Membrane Preparation
}

\author{
H. Manzanarez ${ }^{\mathrm{a}}$, J.P. Mericq ${ }^{\mathrm{a}}$, P. Guenoun ${ }^{\mathrm{b}}$, D. Bouyer ${ }^{\mathrm{a}, *}$ \\ ${ }^{a}$ IEM (Institut Européen des Membranes) UMR 5635 (CNRS-ENSCM-UM2), Université \\ Montpellier, Place Eugène Bataillon, F-34095 Montpellier, France \\ ${ }^{b}$ Université Paris-Saclay, CEA, CNRS, NIMBE, LIONS, 91191, Gif-sur-Yvette, France.
}

\begin{abstract}
Keywords: Phase-field simulation, Cahn-Hilliard model, mobility, membranes, evaporation process
\end{abstract}

\section{Introduction}

Phase separation processes are widely used in industry for manufacturing 3 various types of products, from usual metals to polymer solutions. Prepa4 ration of polymeric membrane is one of these applications of great interest 5 [1, 2]. In the last four decades, research has been dedicated to the polymeric 6 membrane formation mechanisms in order to better control the final mem7 brane morphology [3, 4]. Starting from a homogeneous polymeric solution composed of a polymer dissolved in a good solvent, a thermodynamic demixing process is induced by a temperature change (Temperature Induced Phase Separation or TIPS process) [5] 8] or by the intrusion of a non-solvent of the polymer (Non-solvent Phase Separation or NIPS process) [9, 10]. Starting

\footnotetext{
${ }^{*}$ Corresponding author $U R L$ : denis. bouyer@umontpellier.fr (D. Bouyer)
} 
from a ternary system composed of a polymer, a good solvent and a small amount of a non-solvent of the polymer, a faster evaporation rate of the solvent comparing to that of non-solvent can also induce the phase inversion (Dry-casting process) [11]. During the demixing process, two phases will be created: a polymer-rich phase mainly composed of polymer and a polymerlean phase mainly composed of solvent (and/or non-solvent depending on the process). The polymer-rich phase form the membrane matrix after extraction of the polymer-lean phase which will form membrane pores.

One of most important challenges in membrane manufacturing concerns the control of the final morphology that will strongly affect the membrane performances towards the targeted applications. For instance, asymmetric structures characterized by a pore structure that gradually changes from very large pores to very fine pores at the membrane surface [2], will be targeted for pressure driven membrane processes (filtration in water treatment applications for example). The upper selective layer, responsible for membrane selectivity, should be as thin as possible, while the pore size strongly increases beneath this selective layer to maximize the filtration flux through the membrane. On the contrary, symmetric membranes with uniform structures through the entire membrane thickness could be interesting for applications such as dialysis and electrodialysis, but also microfiltration [12].

Controlling the whole membrane structure is therefore the key point in membrane preparation, but it still remains a goal hard to achieve since the membrane formation mechanisms are quite complex and particularly difficult to simulate, and hence to predict. Phase separation can be described using the equations of Cahn and Hilliard [13] for polymeric systems, where the free 
energy of mixing of the polymeric system is derived from Flory Huggins theory [14] and the mobility term has to be described using a specific equation. In a recent paper, Manzanarez et al. (2017) [15] investigated the influence of this mobility term on the phase separation dynamics for a closed binary polymeric system [16, 17].

However, additional features have to be described to simulate the membrane formation since the phase separation is coupled with transfer phenomena occurring at membrane interfaces. Indeed, mass exchanges often occurs between the membrane and the external environment simultaneously with phase separation. For instance, solvent extraction and non-solvent intake occur during NIPS process [18, 19], while solvent and non-solvent evaporation will be involved during dry-casting process. Recently, we also exhibited how the phase inversion performed by LCST-TIPS process for water soluble polymer systems was coupled with solvent evaporation [20]. Focusing on the modeling of TIPS process, a wide literature exists and various types of models have been developed during the last 30 years (phase field methods, dissipative particle dynamics méthods, Coarse grain simulation, Monte-Carlo simulation...). Caneba and Song [5] were one of the first to develop a 1D phase field model to simulate the TIPS process. They used Cahn-Hilliard equations for spinodal decomposition, Flory-Huggins for thermodynamics and Vrentas models for the description of the mobility terms. Later, Barton and Mc Hugh [16, 21] added a temperature gradient due to heat transport to simulate the droplets growth during demixing process, in 1D geometry yet. Using phase field methods, the impact of a temperature gradient was also investigated by Lee and coworkers [22] in 2D geometry and by Chan [23] in 
1D geometry to better understand the formation of anisotropic morphologies by TIPS process. Later the Cahn-Hilliard equations were solved in 3D geometry for modeling TIPS process [17]. Using different modeling method, He et al. 24], Tang and coworkers simulated the TIPS process by Dissipative Particle Dynamics simulation (DPD) (2013, 2015, 2016) [25 27. Even more recently, Tang and coworkers [28] used DPD and Coarse Grain methods to simulate the coupling between phase separation and mass transfer when the UCST-TIPS process is conducted by immerging a hot polymer solution into a cold water bath. In the latter case, mass exchanges are expected to be very rapid since they occur in liquid phase. However, the coupling between mass exchanges by solvent evaporation and phase separation was less investigated. Mino et al. [17] only considered in their simulations an initial concentration gradient that could be due to an initial solvent evaporation but their simulations did not involve the direct coupling between both phenomena. However, for LCST-TIPS process the coupling between solvent evaporation and phase separation dynamics is crucial since both dynamics are slowed and concomitant [20, 29]. Whatever the process aforementioned, it is of prime importance to elucidate how the mass exchanges affect the phase separation dynamics, and hence the final membrane morphology. Surprisingly, to the best of our knowledge, few theoretical studies have considered this coupling 30.

This paper focuses on the coupling between the phase separation induced by TIPS process and mass transfer phenomena for a simplified binary polymer/solvent system. More specifically, the solvent evaporation occurring at the upper membrane interface will be simulated and its interplay with the 
phase separation dynamics will be investigated.

In the first part of the paper, the coupling between phase separation and solvent evaporation will be simulated and discussed in a horizontal 2D plan, and then in the second part, the simulations will be performed in a $2 \mathrm{D}$ vertical cross-section of the membrane in order to investigate the possible formation of concentration gradients.

Theory

The diffusion equation that describes the phase separation is a modified time-dependent Ginzburg-Landau theory for a conserved order parameter.

$$
\frac{\partial \phi(\mathbf{r}, t)}{\partial t}=\nabla \cdot\left[\Lambda(\phi, \mathbf{r}) \nabla \frac{\delta F}{\delta \phi(\mathbf{r})}\right]+\sigma(\phi, \mathbf{r})
$$

where $\phi(\mathbf{r}, t)$ is the volume fraction of the polymer, $\Lambda(\phi, \mathbf{r})$ the mobility term and $F$ is the free energy functional of the system can thus be expressed as [13] :

$$
F[\phi(\mathbf{r}, t)]=\int d r\left[f(\phi)+\frac{C}{2}|\nabla \phi|^{2}\right]
$$

where $f(\phi)$ is the free energy of mixing per lattice site for polymer solutions described by the Flory-Huggins theory [14]:

$$
f(\phi)=\frac{k_{B} T}{v_{0}}\left[\frac{\phi}{N} \ln \phi+(1-\phi) \ln (1-\phi)+\chi \phi(1-\phi)\right]
$$

were the degree of polymerization $N=150, v_{0}$ is the volume of the monomer and $\chi$ is the interaction parameter. This parameter is supposed to be here an inverse function of temperature. All quenches studied here are made at a constant $\chi$ and are consequently isothermal. $C|\nabla \phi|^{2} / 2$ is the gradient energy contribution which describes the cost of an interface between the two phases 
resulting from the phase separation. The gradient parameter $C$ follow here the Debye approximation [31]:

$$
C(\phi) \approx \frac{k_{B} T}{v_{0}} \frac{\chi}{3} R_{g}^{2}
$$

$R_{g}$ is the radius of gyration of the polymer $\left(R_{g}^{2} \approx a^{2} N / 6\right.$ were $a$ is the Kuhn length and $\mathrm{N}$ the degree of polymerization). The term $\mathrm{C}$ can be related to the polymer chain length and the surface tension. Thus, from a purely numerical point of view, this parameter imposes a space discretization (typically a mesh must be lower than C) which makes it possible to determine the final size of the simulated field $(\mathrm{L}=250 \mathrm{C})$. Finally, the source term $\sigma(\phi, \mathbf{r})$ is added to the continuity equation in order to add evaporative transfers at the interface between the system and the outside.

In a recent paper, we investigated the influence of the mobility term on the phase separation dynamics [15] using Fourier transforms and Minkowski descriptors. Fast, Slow and Vrentas model were compared for various conditions of initial quenching with a polymeric system described by the Flory-Huggins theory. For binary systems, those models were shown to give somewhat different results in terms of growth law. Experimental data found in literature however shown to mostly validate the Fast model [32, 33]. This model was consequently chosen here and writes as:

$$
\Lambda(\phi, \mathbf{r})=\phi(1-\phi)\left[\phi D_{s}+(1-\phi) N D_{p}\right]
$$

In this study, constant values are taken to estimate the tracer diffusion of the solvent $D_{s}$ and the polymer $D_{p}=D_{s} / N$.

To describe the solvent evaporation during phase inversion process, a solvent flux was added at the upper interface, from the polymeric system to 
the external environment:

$$
J_{\text {evap }}\left(\phi, \mathbf{r}_{\text {upper }}\right)=k \Delta g(\phi)
$$

where $\Delta g(\phi)=a_{s}^{i}\left(T^{i}\right)-a_{s}^{\infty}\left(T^{\infty}\right)$ is the difference in activities between the upper polymer solution at the interface and the solvent in the external environment [34 37]. The solvent activity at the upper interface was calculated using the mathematical description of the free energy of mixing, herein the Flory-Huggins theory:

$$
a_{s}=\exp \left[\frac{1}{k_{B} T}\left(\frac{\partial \Delta G_{m}}{\partial n_{s}}\right)\right]
$$

In equation (6), $k$ represents the mass transfer coefficient that mainly depends on the hydrodynamic conditions in the air above the interface and can be calculated using semi-empirical correlations. The evaporation regime are described by the convective Biot number:

$$
B i=\frac{k}{D_{0}} l(t=0)
$$

For the simulations of this work, a simplified approach was used and four different values of $k$ were chosen for testing different regime of evaporation: $B i=0.01,0.1$ and 0.5 . The solvent activity in the external environment was assumed to be null.

It must be noted that the simplified model of evaporation used here is based on the assumption that diffusion of species is the limiting factor of evaporation [38]. This model can be complemented by taking into account the gelification of the polymer in the evaporating crust [34, 39, 40], a phenomenon which is ignored here. Moreover, another approach, alternative to diffusion-limited models, was recently proposed for dealing with non-equilibrium situations [41]. 


\section{Methods}

The non-dimensionnal system of partial differential equations was numerically solved in two dimensions using finite element software: COMSOL Multiphysics 5.4 with the scaling parameters $L_{0}=l_{x}=1[\mu m], t_{0}=L_{0}^{2} / D_{0}$ and the Biot number (equation 8).

A structured moving mesh was used with $64 \times 64$ nodes and it was refined until no change in the numerical result was obtained. A variable time step was used to improve the numerical resolution. For the results in the YZ plane, the resolution of mass transfers process induces a displacement of the upper domain boundary that smoothly displaces the mesh nodes at the surface and inside the bulk of the domain.

The images resulting from phase separation-evaporation simulation were analyzed using Fast Fourier Transform (FFT) performed by Image J (NIST), as well as by calculating the Minkowski descriptors for estimating the topological indicators such as the volume fraction, the connectivity, and the Euler characteristics. More details on the procedure to obtain such data, can be found in our previous paper [15]. For all pictures, the FFT returned a reciprocal space image exhibiting a ring, confirming the existence of a characteristic size of phase separation $L_{m}(t)$. For determining this size with precision, these rings were radially averaged to provide a $I(q)$ curve-also called structure factor- where $\mathrm{I}$ is proportional to the square of the Fourier transform of concentration correlations and $\mathrm{q}$ is the wavevector of reciprocal space. These structure factors were compared at different distances (see below) of the evaporating surface for assessing the phase separation homogeneity.

The thresholding method transforms the grayscale image to black/white 
images by sweeping all thresholds. Then, three topological descriptors are extracted from those images: $\mathrm{F}$ is the ratio of the area occupied by the white pixels divided by the total pixel number, $\mathrm{U}$ is the interface density obtained by counting the pixel number of black/white neighbors and Ec is the Euler characteristic which can be assimilated in this work to the difference between the number of black non-connected domains and the number of white nonconnected domains. All images were normalized in size before analysis to ensure a clear comparison from one image to another

Results

Two sets of results are presented herein: in a first part, simulations were carried out in a 2D XY plane (horizontal plane) as reported in Figure 1. In a second part, simulations in a $2 \mathrm{D}$ vertical $\mathrm{YZ}$ plane are presented to simulate the phase separation in the membrane cross-section.

Evaporation simulations in a horizontal XY $2 D$ plane

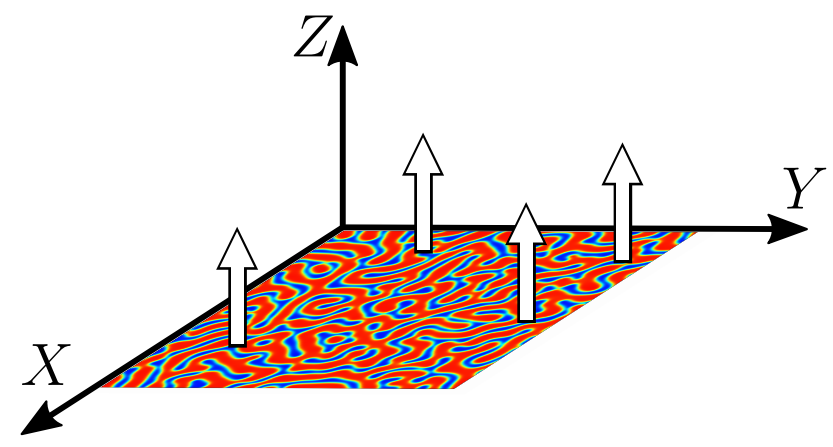

Figure 1: Schematic representation of the evaporation process in the XY plane

These first sets of simulations in the horizontal XY plane aimed at testing a solvent loss in a 2D domain of $1[\mu m] \times 1[\mu m]$ where each point of the sim- 

solvent evaporation.

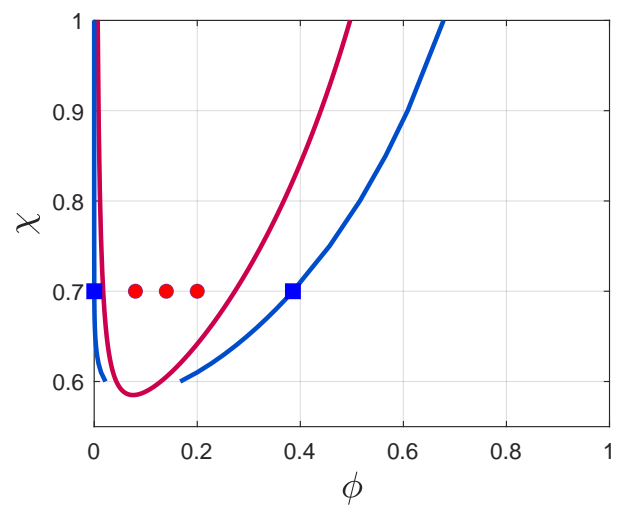

Figure 2: Phase diagram for a asymmetrical system $(N=150)$. The blue and red curves represent the binodal and the spinodal curve, respectively. The red point represent the initial composition tested in this study. The blue square gives the binodal phase composition equilibium. In any spatial point of the phase field, The composition path goes from a red circle to $\phi=1$. For separation in the XY plane, the leftmost red point was chosen as the quenching point ( $\mathrm{phi}=0.08)$.

ulation domain was affected by the solvent loss (Figure 1). This 2D domain could conceptually correspond to the upper interface of a membrane exposed to air, as if the membrane would have null thickness. In this respect, bulk phenomena occurring deeper in the membrane were not taken into account for those first sets of simulations.

Considering the boundary conditions, the solvent activity in the air was considered to be null, assuming continuous ventilation in the external environment above the polymer solution, hence maximizing the driving force for 
this quenching point was $\phi_{i n i t}=0.08$ for this separation in the XY plane.

Starting from this phase diagram (Figure 2) and this quenching point, in a closed system the phase separation would lead to the formation of a continuous polymer-lean phase and a disperse polymer-rich phase. At equilibrium, each phase would be expected to tend to its equilibrium concentration: $\phi_{a}=1.4 \cdot 10^{-4}$ for the polymer-lean phase and $\phi_{b}=0.386$ for the polymerrich phase (cf. Figure 2). In absence of evaporation, the global polymer volume concentration $\phi$ is constant, equal to 0.08 and the volume fractions of each phase keep constant values, equal to $\phi_{\text {rich }}=0.21$ and $\phi_{\text {lean }}=0.79$, respectively, as calculated by the lever rule.

Now, when considering the coupling between phase separation and solvent evaporation, the global polymer concentration is expected to gradually increase in the system in such a way that the system follows a composition path that will ultimately reach the right of the phase diagram, following the dotted line at constant $\chi=0.7$ (Figure 2).

For the first set of simulations in the 2D XY plane, the nondimensional mass transfer coefficient $k$ was fixed at 0.1 . In Figures 3 a-j, the patterns obtained in a closed system (upper row, without evaporation) and in presence of continuous solvent evaporation (lower row) were compared. Without solvent evaporation (Figures 3 a-e), a spontaneous phase separation starts with the formation of droplets of polymer-rich phase into a continuous polymer-lean phase. The concentration of the polymer-rich phase tends to $\phi_{\text {rich }}^{e q}=0.386$ while the concentration of polymer-lean phase tends to $\phi_{\text {lean }}^{e q}=1.4 \cdot 10^{-4}$ but these concentrations are not reached within the simulation timeframe, as shown by Minkowski descriptors hereafter. Figures 3 f-j represent the simula- 


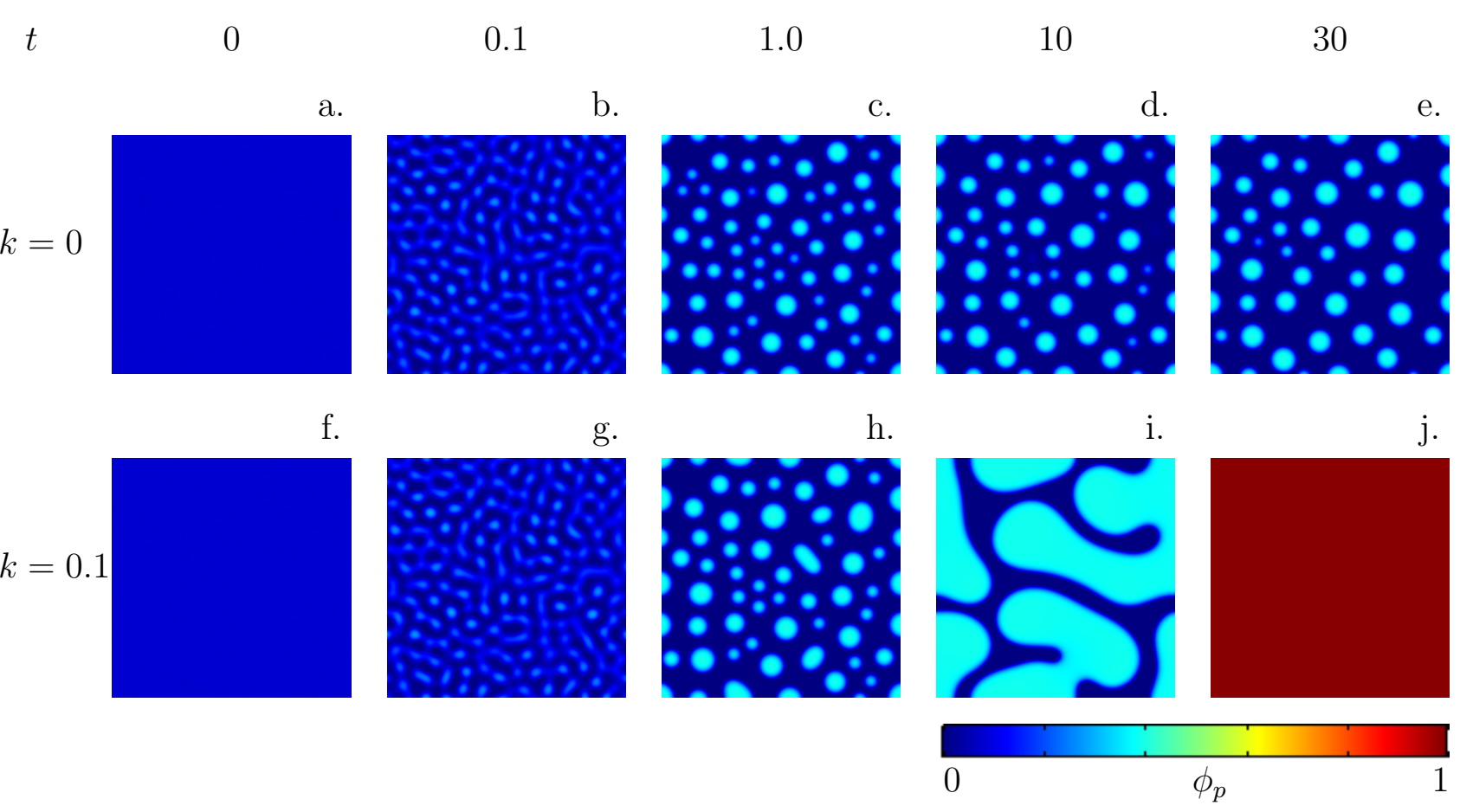

Figure 3: Time evolution of the patterns obtained in closed system (a-e) and with a coupling between phase separation and solvent evaporation (f-j). $\phi$ is the polymer concentration.

tion run corresponding to the coupling between phase separation and solvent evaporation. The same initial quenching point $\chi=0.7$ and initial polymer concentration $\phi_{\text {init }}=0.08$ were chosen for comparison.

Figures 3 f-j clearly exhibit how the solvent evaporation affects the phase separation dynamics. The solvent loss leads to a displacement along a composition path to the right of the phase diagram, and leads to an increase in the volume fraction of the polymer-rich phase. The system undergoes a percolation inversion between Figure $3 \mathrm{i}(t=10)$ and $3 \mathrm{j}(t=30)$ and in the same time, the continuous increase of the global polymer volume frac- 
tion promotes the coalescence of the rich phase droplets. Around $t=10$ a bicontinuous polymer-rich phase is formed coexisting with a bicontinuous polymer-lean phase although very dissymetrical. The percolation inversion leads to the formation of droplets of polymer-lean phase in a continuous polymer-rich phase. Later, on the composition path leaves the diphasic region, leading to the formation of a continuous phase, highly concentrated in polymer (Figure $3 \mathrm{j}$ ).

In a previous paper [15], we analyzed the patterns using both Fourier transform and Minkowski descriptors. The latter method was used in this work to analyze more deeply the influence of the coupling between solvent evaporation and phase inversion dynamics: the patterns were binarized using a chosen threshold, and then the binarized images were analyzed with three Minkowski descriptors: volume fraction, connectivity, Euler characteristics.

The use of Minkowski descriptors requires performing a prior binarization of the patterns. The choice of the threshold is not trivial since it could significantly affects the curves interpretation. In our previous paper, the binarization threshold was chosen equal to the initial polymer concentration in such a way that during the phase separation, the regions characterized by higher polymer concentration than the initial polymer concentration were represented in white color, while the regions with lower concentrations than the initial polymer concentration were represented in black color. In this way, it was easy to catch the formation of the polymer-lean and polymer-rich phases as soon as the demixing process started [15]. Although the problem is different in this work since the solvent evaporation induces a continuous increase of the polymer concentration, the same thresholding procedure was 
a.

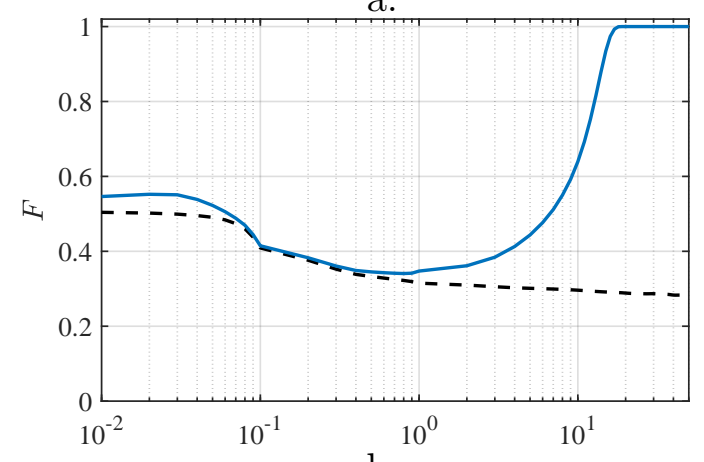

b.

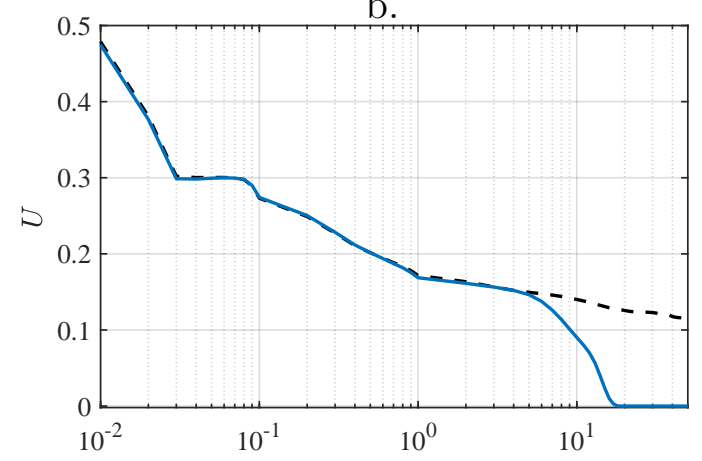

c.

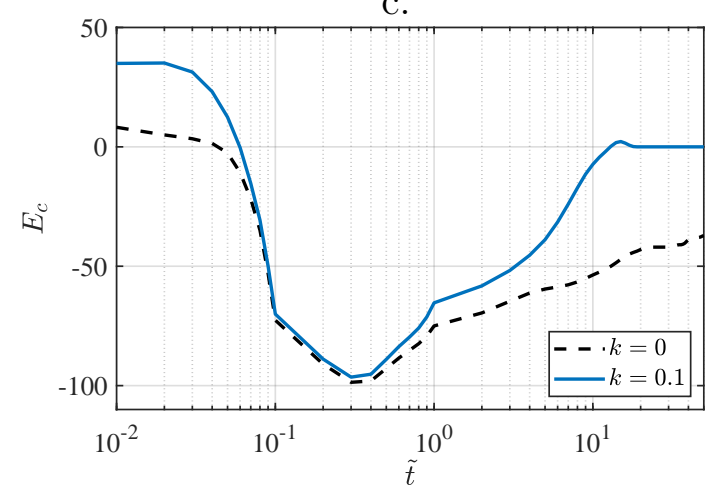

Figure 4: Time evolution of the Minkowski descriptors in closed system (dash line) and for the coupling between phase inversion and solvent evaporation (blue line) for $k=0.1$. a. represents the variation of the covered area $F$ occupied by the rich phase with time, b. the interface density $U$ and c. the Euler characteristic $E_{c}$. 
chosen 1

The binarization threshold was thus fixed at a polymer concentration of 0.08 for the Minkowski analysis presented in Figure 4. In this context, the polymer-rich phase corresponds to regions where $\phi_{p}>0.08$ and the polymer-lean phase corresponds to regions where $\phi_{p}<0.08$. Following this procedure, it should be noted that a fraction of the interfaces was counted as polymer-rich phase since the interface is characterized by a polymer concentration gradient between the polymer-rich and polymer-lean concentrations $\left(\phi_{\text {rich }}^{e q}=0.386\right.$ and,$\phi_{\text {lean }}^{e q}=1.4 \cdot 10^{-4}$ respectively, when the concentrations tend toward the equilibrium concentrations). The three Minkowski descriptors were calculated and reported in Figure 4 versus time for the closed and the open system with $k=0.1$.

Figure 4.a represents the variation of the covered area occupied by the rich phase with time. Without evaporation the volume fraction of each phase is not expected to change during demixing process, so the Minkowski descriptor $\mathrm{F}$ is shown to tend towards $F_{\infty}=0.21$ with regards to the level rule. After $t=50$, at the end of the simulation, Figure 4. a exhibits that the system is almost at equilibrium in terms of volume fraction of each phase ( $\mathrm{F}$ is close to $0.21)$. On the contrary, when the evaporation is coupled to the phase separation, the system is continually in non-equilibrium state and the dynamics of

\footnotetext{
${ }^{1}$ Actually, as shown by volume fraction temporal evolution, the average domains concentration is very quickly different from the initial value : this latter choice is then still suitable for defining separating domains. However, as the interface is rather steep between phases, the error is believed to be negligible since 0.08 is far from actual concentrations of evaporating phases
} 
phase separation is different. Due to continuous solvent loss, the descriptor F gradually increases until reaching unity, corresponding to pure polymer, around $t=20$. Note that no slope change was observed once the composition path passes through the binodal curve, corresponding to a polymer concentration close to 0.386. At this time (around $t=20$ ), the system is composed of a continuous phase characterized by a high concentration in polymer with a covered area fraction of 1 .

As reported in a previous paper [15], $\mathrm{U}$ is the interface density (boundary length in 2D) and Ec is the Euler characteristic, useful for analyzing the connectivity of domains. Without evaporation, Figure 4. b exhibits that the boundary length $\mathrm{U}$ continuously decreases. The small droplets formed at initial stage are expected to grow and they coalesce with other droplets or disappear due to ripening effect, thus decreasing the total interface length. In presence of solvent evaporation, the curve of the interface density $\mathrm{U}$ shows the same trend as the curve without evaporation during the first time steps, and then the interface density $U$ is shown to decrease steeper until zero due to the disappearance of solvent droplets.

Without solvent evaporation, the Euler characteristic $E_{c}$ en Figure 4.c was shown to sharply decrease at the beginning of the phase separation because of the creation of numerous dispersed droplets, and then $E_{c}$ slightly increases due to the reduction of the droplets number. The Euler characteristics $E_{c}$ was shown to keep negative values in a closed system, indicating the absence of percolation inversion: the polymer-rich phase is always the dispersed phase into a continuous polymer-lean phase. On the contrary, the Figure 4.c exhibits that a percolation inversion is detected using this descrip- 
tors. Now the Euler characteristics reaches positive values when the solvent evaporation takes place, which indicates that the percolation inversion occurred around $t=12$. Then, droplets of polymer-rich phase disappeared and were rapidly replaced by droplets of polymer-lean phase in a polymerrich continuous phase. The main interest of the analysis using Minkowski descriptors lies in the fact that the time of the percolation inversion can be detected with a good precision without the necessity to observe the patterns, which represents a significant time savings.

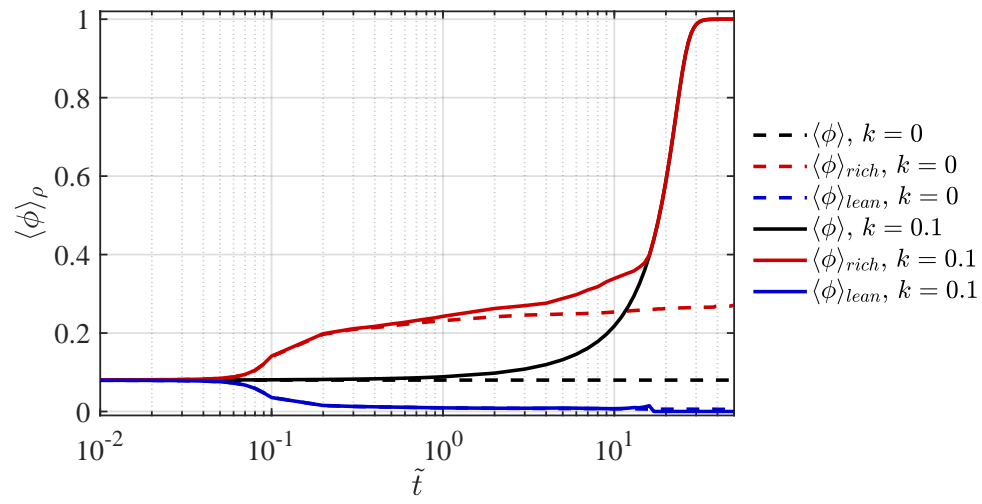

Figure 5: Variation of the global concentration (black curves), polymer concentration in the polymer-lean phase (blue curves) and polymer-rich phase (red curve). Dashed lines correspond to the closed system and solid lines correspond to the case with solvent evaporation.

To keep on analyzing how the solvent evaporation affects the phase separation dynamics, we reported the composition of the polymer-lean and polymer-rich phases averaged on domains during the demixing process (Figure 5). The binarization threshold was fixed at 0.08 . The dashed lines correspond to the case without evaporation and the solid lines to the case with 
solvent evaporation. The black, red and blue curves correspond to the global polymer concentration in the whole domain, the polymer concentration in the polymer-rich phase and the polymer concentration in the polymer-lean phase, respectively. For instance, for the closed system (without evaporation) the mean polymer concentration (black dotted line) is constant, equal to the initial concentration $\left(\phi_{\text {init }}=0.08\right)$. The polymer concentrations in each separated phase tend toward the equilibrium concentration $(0.386$ for the rich phase and $1 \cdot 4 \cdot 10^{-} 4$ for the lean phase). It is shown that the concentration in the lean-phase is rather close from the equilibrium final concentration while the concentration in the polymer-rich phase is still quite distant from the final value since the separation was started at 0.08 .

When coupling solvent evaporation and phase separation (solid curves), the mean polymer concentration continuously increases, in agreement with the displacement of the composition path in the phase diagram toward higher polymer concentrations. For the lean phase, the curve is slightly above the curve corresponding to the closed system until around $\tilde{t}=12$, which suggests that the relaxation dynamics due to the phase separation is too slow to compensate the continuous solvent loss. In other words, the system is continuously forced to be in non-equilibrium state because of solvent evaporation.

The same conclusion can be drawn considering the polymer-rich phase: the solvent evaporation leads to a faster increase of the polymer-rich phase concentration. In the polymer-rich phase too, the relaxation time scale of the phase separation is clearly slow enough to evidence the solvent evaporation.

This first simulation run conducted in horizontal 2D plane exhibited how the solvent evaporation affects the phase separation dynamics, leading to an 
Evaporation simulations in a (YZ) $2 D$ plane

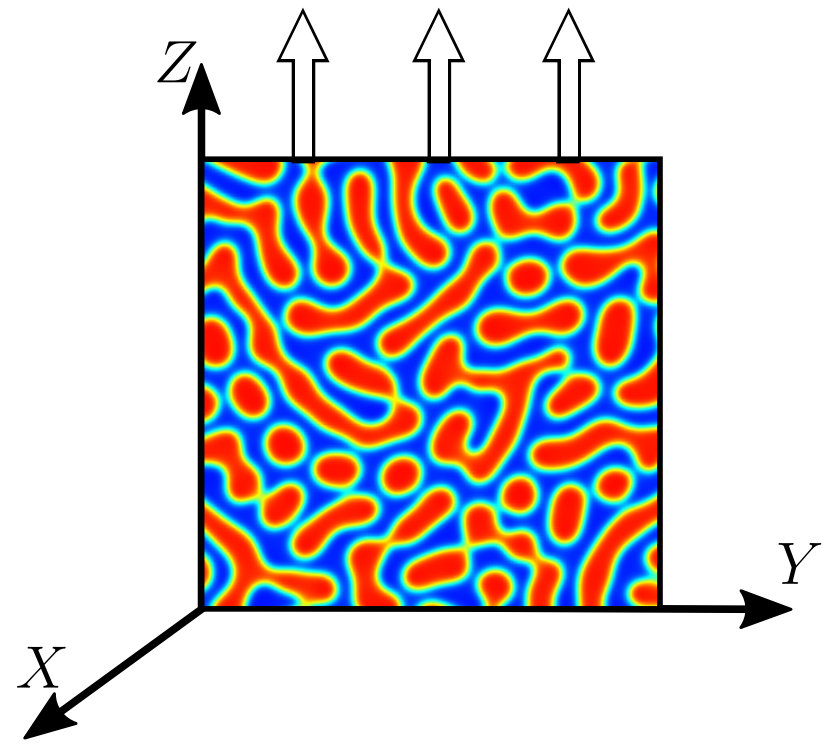
$\phi_{\text {init }}=0.08$ and then to the formation of a monophasic system when the composition path goes through the binodal line of the phase diagram.

Figure 6: Schematic representation of the evaporation process in YZ plane

inversion percolation when starting at an initial polymer concentration of

Figure 6: Schematic representation of the evaporation process in $Y Z$ plane

Another set of simulations were performed in a vertical YZ plane as represented in Figure 7. The 2D plane can now be assimilated to the cross-section of the system of $1[\mu \mathrm{m}] \times 1[\mu \mathrm{m}]$. The bottom of the system at $Z=0$ is closed, hence assuming no mass exchange, whereas the upper-layer (coordinate $Z=L$ at $t=0$ ) is assumed to be in contact with the external environment in such a way that solvent evaporation can occur. Periodic boundary conditions were imposed at the left and right boundaries of the system. The global mass balance was calculated at each time step assuming density con- 
servation and the thickness $l(t)$ of the $\mathrm{YZ}$ domain was expected to decrease because of solvent loss assuming the following equation:

$$
\frac{d l}{d t}=J_{\text {evap }}
$$

$J_{\text {evap }}$ is determined by the flux of solvent, expressed by the equation (7). Simulations were performed assuming three different quenching points at three initial polymer concentrations $\left(\phi_{\text {init }}=0.08,0.14\right.$ and 0.20 , respectively), and four nondimensional values of the mass transfer coefficient $\mathrm{k}$ that correspond at the Biot number $(B i=0.01, B i=0.1$ and $B i=0.5)$.

At early times, a surface directed phase separation is evidenced due to breaking of symmetry caused by evaporation. This is evidenced as a surface composition wave limited in extension to a few wavelengths (see top of figures 7 at $\mathrm{t}=1$ where horizontal domains are evidenced) and whose wavelength value is close to the bulk phase separation characteristic length.

These surface directed phase separation patterns have been evidenced both experimentally due to preferential wetting constraints [42] or theoretically because of thermal gradients on one side [4] or, more recently, because of solvent replacement in a ternary solution [30]. The evaporating surface can be viewed as a wetting constraint breaking isotropy, contrary to the neutral sides and bottom surfaces where isotropy is maintained by periodic boundary conditions and Dirichlet condition respectively.

At later times, when the surface directed phase separation wave has disappeared, a dense layer is formed in the vicinity of the upper interface.

In Figure 8 are reported the patterns obtained for the three initial quenching points, without evaporation and with solvent evaporation for the interme- 


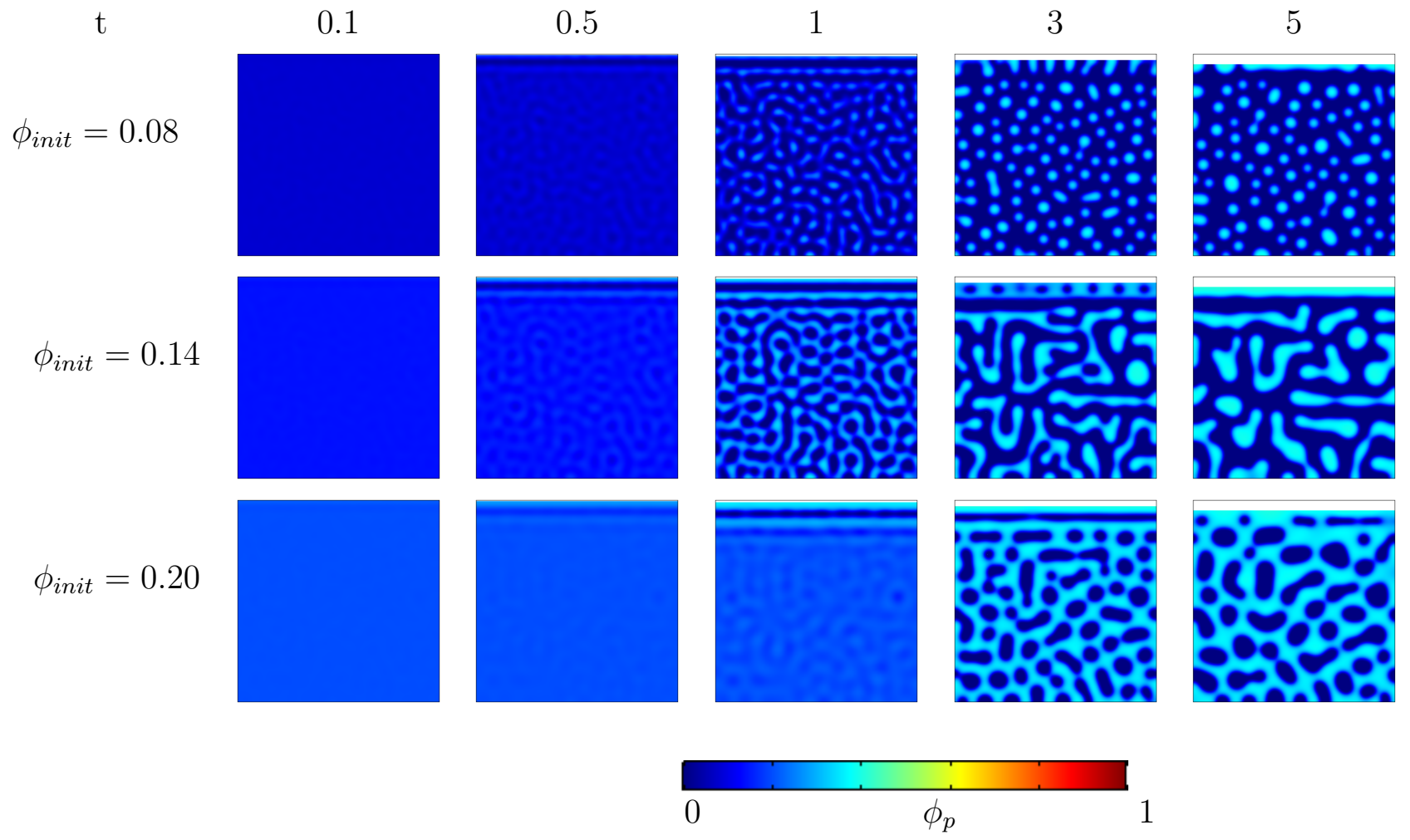

Figure 7: Early times phase separation patterns under evaporation of solvent by the top most surface for $B i=0.1$. A surface composition wave is established more rapidly than the bulk PS. This wave eventually transforms in the top skin layer which is characteristic of late times evolution.

diate evaporation rate $(B i=0.1)$ at late times $(t>=6)$. With evaporation, the formation of a dense layer and the decrease of the total height were clearly observed, whatever the initial polymer concentration $\phi_{\text {init }}$.

When the initial polymer concentration $\left(\phi_{\text {init }}\right)$ is lower than 0.14 , droplets of polymer-rich phase are dispersed into a continuous polymer-lean phase. Besides, symmetrical interconnected phases are observed when $\phi_{\text {init }} \approx 0.14$ and droplets of polymer-lean phase are dispersed into a continuous polymer- 
rich phase when $\phi_{\text {init }} \approx 0.20$. In all cases the dense phase (or skin) on top is clearly inhomogeneous along $\mathrm{z}$ and forms a quasi-planar interface with the phase-separating region below for $\phi_{\text {init }}=0.08$ whereas the skin is continuously linked with the phase-separating region in the other cases.

A qualitative visual observation of the snapshots suggests that the formation of the upper dense polymer layer weakly affects the dynamics of phase separation beneath the dense layer: the patterns are very similar with and without the solvent evaporation. The simulations launched with $\phi_{\text {init }}=0.14$ that show interconnected structures, exhibit slight differences near the dense layer region. At $\phi_{\text {init }}=0.08$ and $\phi_{\text {init }}=0.20$ the differences are even weaker with and without solvent evaporation (see below Figure 12 for a quantitative analysis).

To refine the previous observations, we reported in Figure 9 the patterns obtained at $t=1$ of the twelve simulations (3 concentrations, 4 evaporation rates). The first, second and third lines correspond to quenching point at initial concentrations $\left(\phi_{i n i t}=0.08\right), \phi_{i n i t}=0.14$ and $\phi_{\text {init }}=0.20$, respectively. The evaporation rate taken into account for those simulations were $B i=0.01, B i=0.1$ and $B i=0.5$ a, for columns 1,2 and 3 respectively.

Not surprisingly, increasing the mass transfer coefficient leads to faster evaporation, to a faster decrease of domain height. A dense layer is formed which seems thicker when increasing not only the initial polymer concentration, but also the mass transfer coefficient Bi.

The dense layer is fairly easy to define when the continuous phase is the polymer-lean phase (case for $\phi_{\text {init }}=0.08$ ) since an interface is clearly formed. For the other cases $\left(\phi_{\text {init }}=0.08\right.$ and $\left.\phi_{\text {init }}=0.20\right)$, the dense layer 


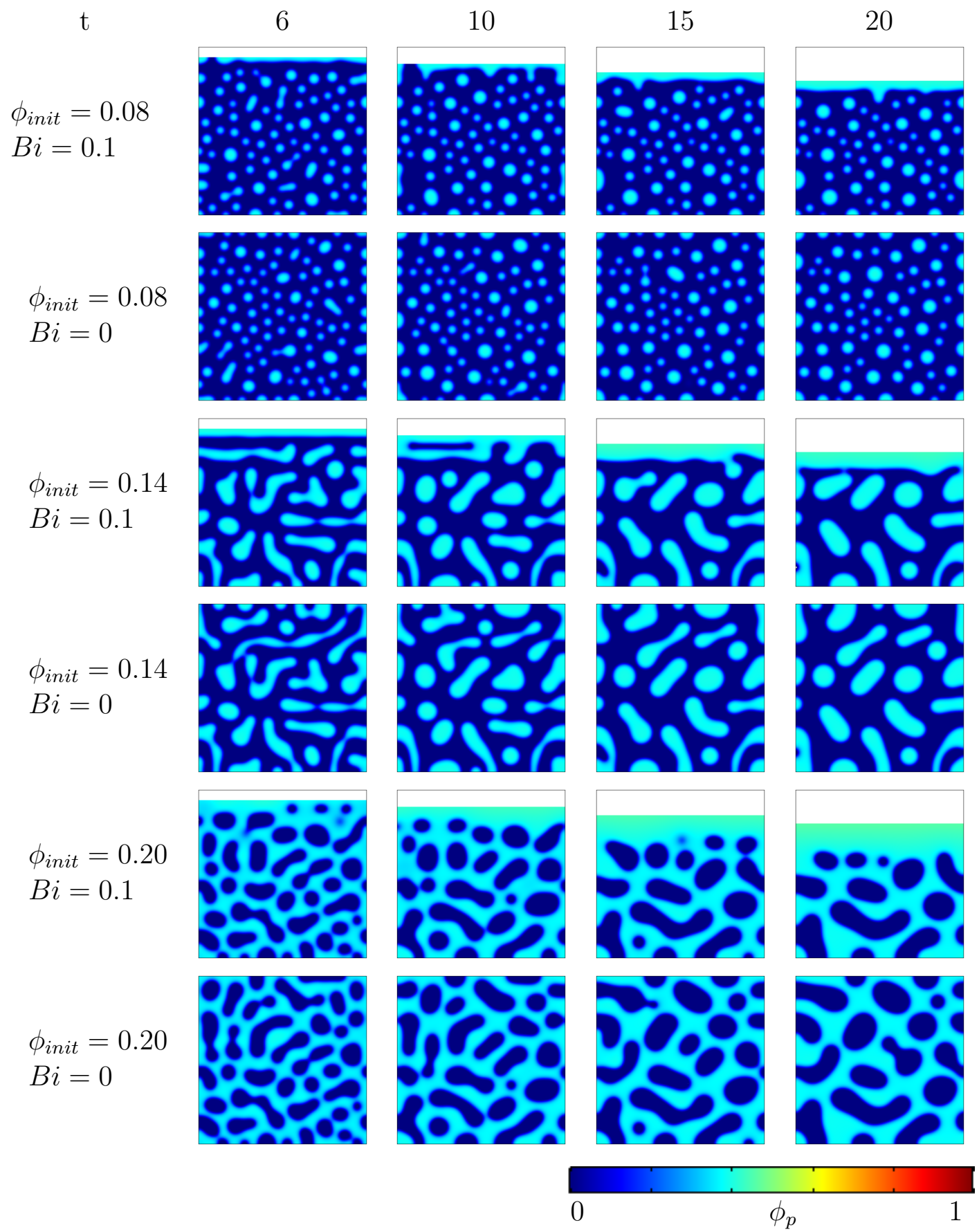

Figure 8: Patterns obtained at increasing time steps for different values of $\phi_{\text {init }}$ with $B i=0.1$. 
thickness should be carefully evaluated (Figure 9). We decided to define the lower boundary of the dense layer as the Z-coordinate where the polymer concentration reaches the equilibrium concentration (0.386): actually a concentration larger than 0.386 is very quickly reached as soon as the thick layer is detectable. The dense layer thickness was reported in Figure 10. b for the aforementioned initial conditions of simulation $\left(\phi_{\text {init }}=0.08,0.14\right.$ and 0.20 and $B i=0.1$ at $\mathrm{t}=2$ ).

As visible in Figure 9, the thickness of the dense layer increases more rapidly at higher initial polymer concentration $\left(\phi_{\text {init }}=0.20\right)$. This is due to the fact that at higher initial polymer concentration, the binodal of the dense phase is reached earlier compared to lower initial polymer concentration when solvent evaporation occurs. Since the lower interface of the dense layer was not perfectly flat in the simulated patterns due to the phase separation and the presence of droplets or interconnected structures, its thickness was estimated using an average along the Z-axis. The difficulty of identifying the dense layer is exemplified by the wavy curve shape in Figure 10,b, especially when droplets of polymer-lean phase are dispersed in a continuous phase of polymer-rich phase $\left(\phi_{\text {init }}=0.20\right)$.

Below the dense (skin) layer, a visual observation of the polymer-rich and polymer-lean phases indicates that no gradient exist in polymer concentration in both phases. In other words, the patterns presented above suggest that the relaxation dynamics below the skin layer are sufficiently fast to maintain the polymer-rich and polymer-lean phases close to the equilibrium values despite the solvent evaporation at the upper interface.

In order to better evidence this absence of gradients, we report in Figure 


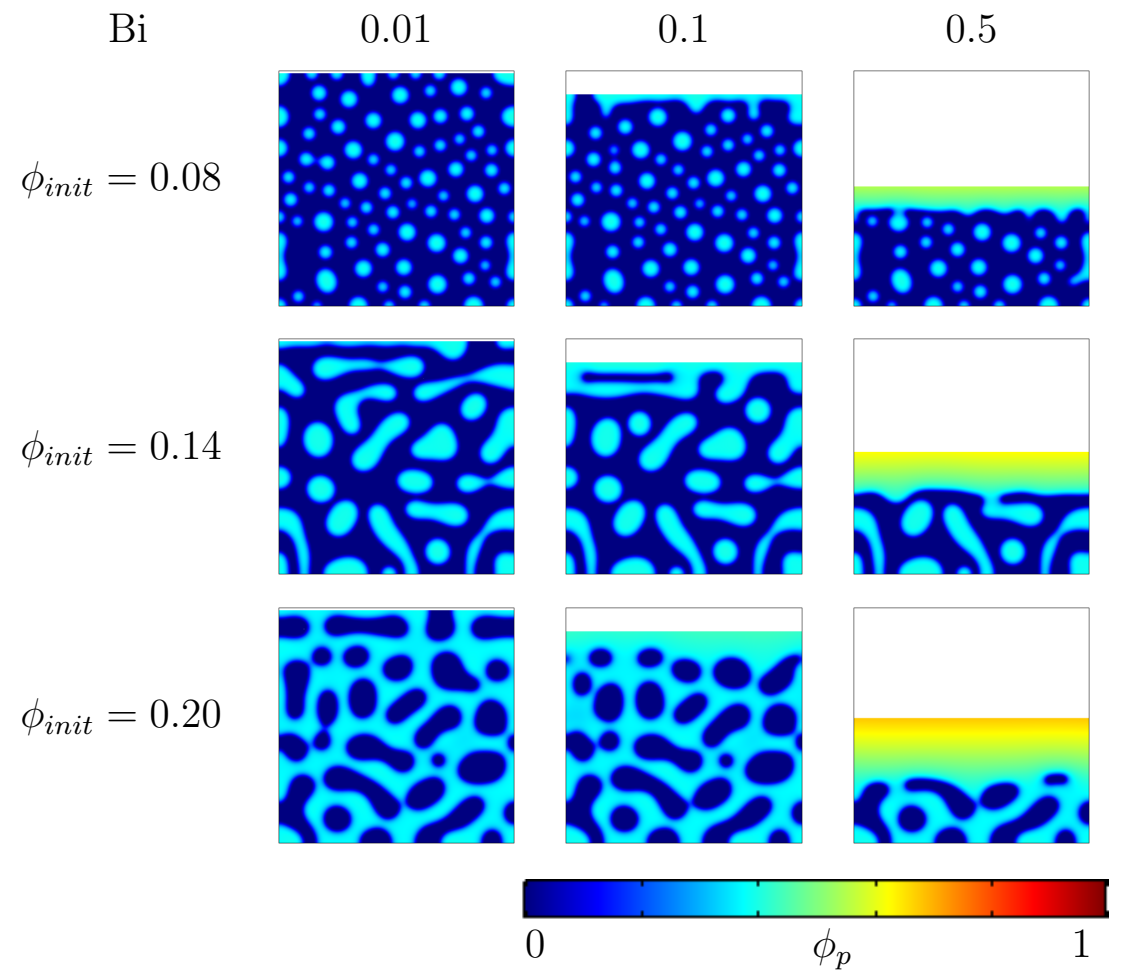

Figure 9: Patterns obtained at different initial polymer concentrations $\phi_{\text {init }}$ and different values of the mass transfer coefficients Bi for $t=1$.

10 the patterns at the time step $(\mathrm{t}=2)$ after having performed a specific thresholding (note that it is not a usual binary thresholding i.e. black and white):

- to focus on the polymer-rich continuous phase (images a), b) and c) in Figure 10], a threshold was fixed at $\phi_{s 1}=\phi_{b}-0.05=0.33$ where all concentrations lower than $\phi_{s 1}$ are assigned to a white color (inverse thresholding) whereas the color code is respected for $\phi>0.33$. As a result, a concentration gradient is visible in the dense layer but not beneath it, i.e. in the diphasic region 
a.

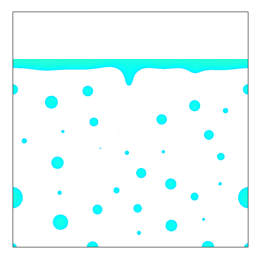

d.

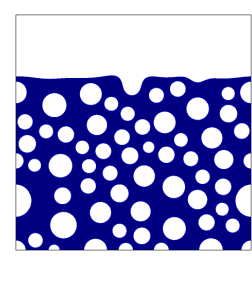

b.

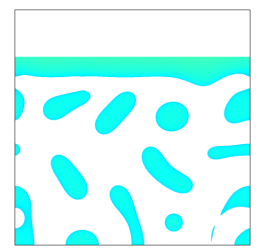

e.

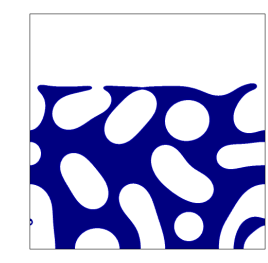

c.

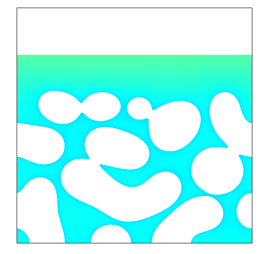

f.

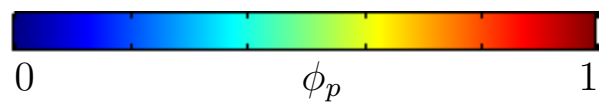

Figure 10: Bulk polymer concentration following the color code in the concentrated phase $(\mathrm{a}), \mathrm{b}), \mathrm{c})$ ) and the lean phase (d),e), f)) respectively for different quenching concentrations: left $\phi_{\text {init }}=0.08$, middle $\phi_{\text {init }}=0.14$ and right $\phi_{\text {init }}=0.20$ at $\mathrm{t}=2 \mathrm{~s}$. Thresholds are chosen to render the lean (resp. the concentrated phase) white to reveal the other one (see text)

- to focus on the polymer-lean continuous phase (images d), e) and f) in Figure 10), a threshold was fixed at $\phi_{s 2}=\phi_{a}+0.05 \approx 0.05$ in such a way that when the concentration exceeded 0.05 , its value was fixed to a white color. On the contrary, for concentrations between 0 and 0.05 , the color scale is respected and exhibits the absence of color gradient, i.e. of concentration gradient in the z-direction in the polymer-lean phase.

The Z-average concentrations over the continuous phase were calculated along a vertical Z line at each Y-coordinate (Figure 11). the plots confirms that below the dense layer and at $t=2$, the concentration in the polymer- 


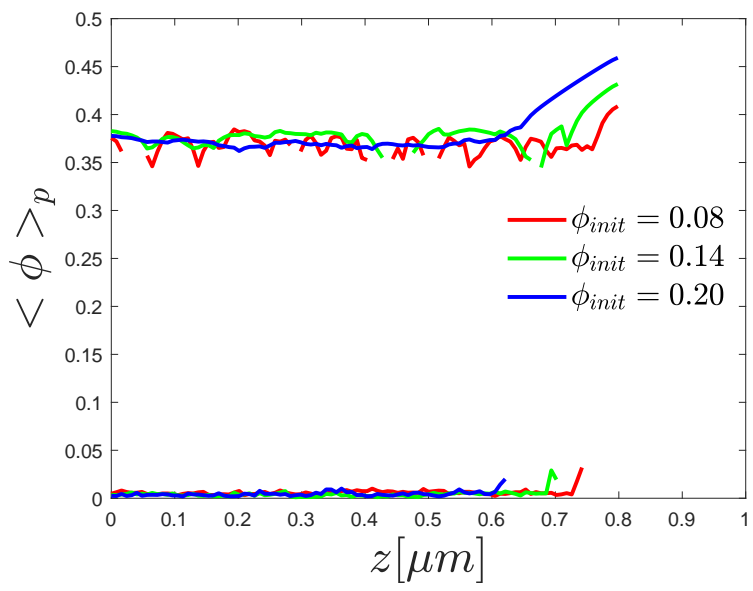

Figure 11: Z-average concentrations over the continuous phase for the three different concentrations after the thresholding procedure of Figure 10.

lean phase for $\phi_{\text {init }}=0$ is very close to $1.10^{-4}$ and the concentration in the polymer-rich phase for $\phi_{i n i t}=0.37$ is also very close to 0.386 . No concentration gradient was observed from the bottom to the dense layer interface, whatever the case.

To complete the quantitative image analysis, 2D Fourier transform (FFT) were calculated for the images to check to what extent the evaporation did affect the phase separation dynamics. For the three pictures at the latest times of separation and the three different initial concentrations, the structure factors were calculated in two rectangular windows. Each window is rectangular of width 446 pixels and height 152 pixels and one is located close to the interface and the other down close to the lower border (see Figure 12 for details). Structure factors are shown to be similar close and far to the interface, proving homogeneity. In particular, for each pair of curves at equal time and concentration, a peak at small wavevectors is evidenced de- 
spite the large typical distance between domains which pushes the peak to the y-axis. This peak is however distinguishable and representative of the distance between domains, which is similar at the top and bottom.

To summarize the previous simulation results obtained in the YZ place, we exhibited that the continuous solvent loss at the top surface due to evaporation induces the formation of a skin layer (note here that no change of dynamics such as gelation or glass transition was assumed to take place in this layer [36]), which suggests that mass transfer localized at the upper interface is faster than the potential inflow of solvent from deeper layers by molecular diffusion. The system relaxes to minimize its free energy in such a way that an equilibrium is reached between the lower part of the dense layer and the adjacent bottom separating layer composed of polymer-lean phase or polymer-rich phase. The thickness of the gradient zone was shown to increase during time whatever the Biot number, i.e. the relative evaporation rate driven by the air flow conditions. In this way, beneath this dense layer, the phase separation in the bulk solution was shown not to be affected by the mass transfer occurring at the upper system interface.

\section{Conclusion}

Herein, we developed a model that coupled the demixing process and the solvent evaporation during the membrane formation by TIPS process. Simulations have been performed in a 2D geometry in XY plane (membrane surface) and YZ plane (cross-section). The simulations in the X-Y plane clearly predicts the existence of an evaporation regime where an initially minority phase rich in polymer will be turned into a majority phase (percolation inversion). This is confirmed in Y-Z simulations where a "polymer 

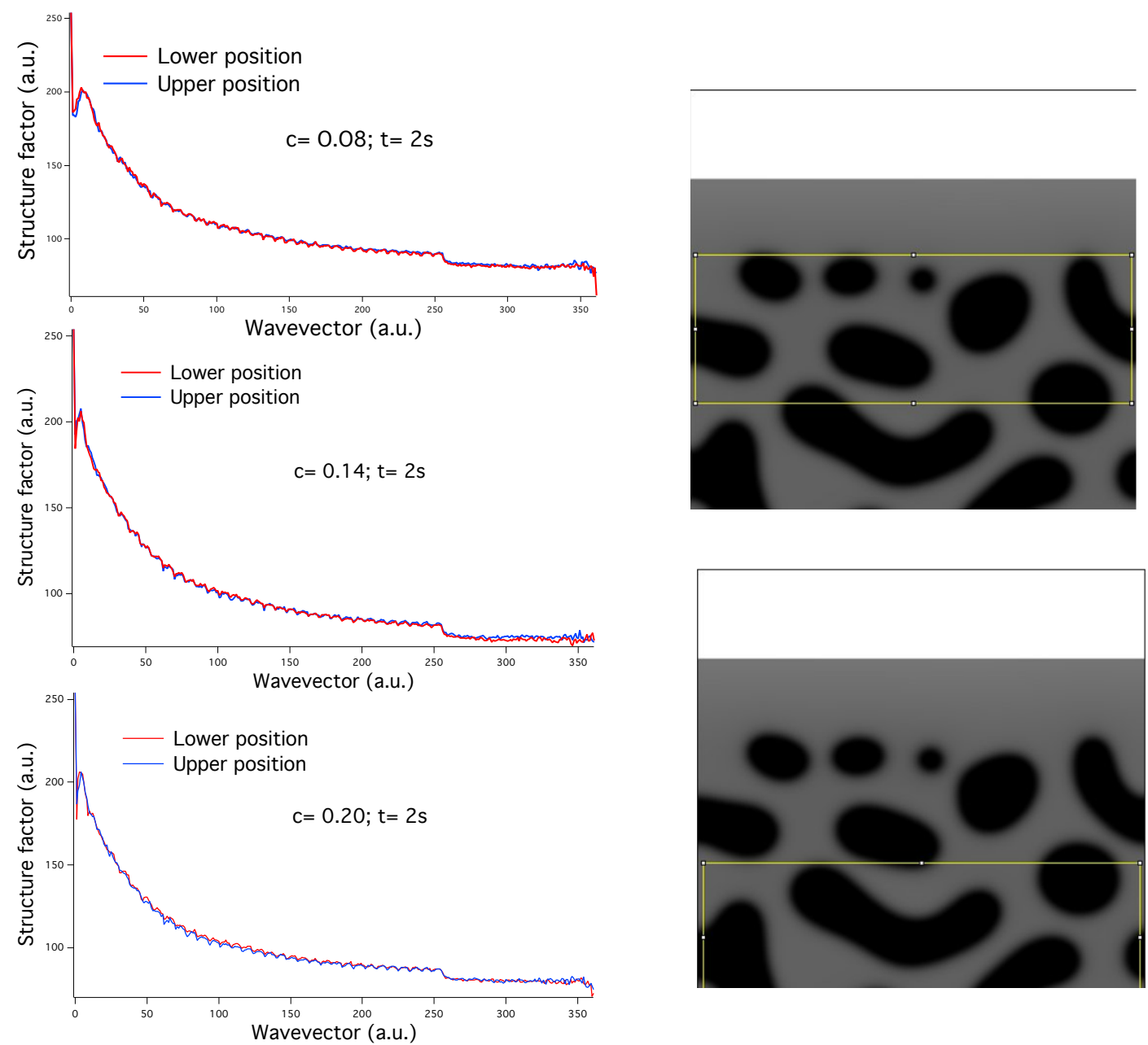

Figure 12: (left) Superposition of structure factors at two different distances from the dense evaporating layer and for the three concentrations at the latest time studied. Oscillations are non-physical and merely due to the pixellisation inhomogeneity whereas the dip at a wavevector of ca. 251 is due to the sudden drop in analyzed pixels number above the maximum inscribed circle radius. (right) Position of the chosen analysis rectangles. 
skin layer" appears after some time on top of an initially dilute phase. Surprisingly and interestingly enough, our results predict that the skin layer is a gradient zone in concentration between a value that tends to one at the top surface and the equilibrium value of the polymer-rich phase which is maintained throughout the evaporation process. This result demonstrates that beneath the skin layer, the phase separation was not affected by the solvent loss at the top surface and stays homogeneous through the entire bulk volume, which was not expected a priori. In this context, the simulation results presented in this work allow a better understanding of the interplay between the solvent evaporation and demixing process, especially for predicting the skin layer thickness, which depend on the evaporation rate. The formation of this skin layer (whose porosity is often facilitated by porogen additives in industry) is of crucial importance for the membrane preparation since it controls the membrane selectivity. The thickness of this layer also plays an important role in the membrane permeability since the main resistance to mass transfer is localized in it. Furthermore, the model provides an insight on the interplay between the solvent evaporation and the demixing process deeper in membrane. Indeed, this work demonstrated that the dynamics of phase separation below the skin layer was not affected by the solvent evaporation, meaning that the pore size within the membrane bulk is not affected by the solvent evaporation. This suggests that the global membrane porosity (the void ratio) would not be affected by the solvent evaporation. On the theoretical side, our results are foreseen to be extended to a tridimensional geometry for coupling $\mathrm{X}-\mathrm{Y}$ and $\mathrm{Y}-\mathrm{Z}$ processes. 


\section{References}

[1] P. van de Witte, P. Dijkstra, J. van den Berg, J. Feijen, Phase separation processes in polymer solutions in relation to membrane formation, Journal of Membrane Science 117 (1) (1996) 1 - 31. doi:http://dx.doi.org/10.1016/0376-7388(96)00088-9. URL http://www.sciencedirect.com/science/article/pii/ 0376738896000889

[2] M. Ulbricht, Advanced functional polymer membranes, Polymer 47 (7) (2006) 2217 - 2262, single Chain Polymers. doi:https://doi.org/10.1016/j.polymer.2006.01.084. URL http://wWw.sciencedirect.com/science/article/pii/ S0032386106001303

[3] R. Kesting, Synthetic polymeric membranes: a structural perspective, Wiley, 1985.

[4] M. Mulder, Basic Principles of Membrane Technology, Springer Netherlands, 1996.

[5] G. T. Caneba, D. S. Soong, Polymer membrane formation through the thermal-inversion process. 1. experimental study of membrane structure formation, Macromolecules 18 (12) (1985) 2538-2545. arXiv:http:// dx.doi.org/10.1021/ma00154a031, doi:10.1021/ma00154a031. URL http://dx.doi .org/10.1021/ma00154a031

D. R. Lloyd, K. E. Kinzer, H. Tseng, Microporous membrane formation via thermally induced phase separation. i. solid- 
liquid phase separation, Journal of Membrane Science 52 (3) (1990) 239 - 261, selected papers presented at the Third Ravello Symposium on Advanced Membrane Science and Technology. doi:https://doi.org/10.1016/S0376-7388(00)85130-3.

URL http://www.sciencedirect.com/science/article/pii/ S0376738800851303

[7] D. R. Lloyd, S. S. Kim, K. E. Kinzer, Microporous membrane formation via thermally-induced phase separation. ii. liquid-liquid phase separation, Journal of Membrane Science 64 (1) (1991) 1 - 11. doi:https://doi.org/10.1016/0376-7388(91)80073-F. URL http://www.sciencedirect.com/science/article/pii/ $037673889180073 \mathrm{~F}$

[8] M. Shang, H. Matsuyama, M. Teramoto, D. R. Lloyd, N. Kubota, Preparation and membrane performance of poly(ethyleneco-vinyl alcohol) hollow fiber membrane via thermally induced phase separation, Polymer 44 (24) (2003) 7441 - 7447. doi:https://doi.org/10.1016/j.polymer.2003.08.033. URL http://wWw.sciencedirect.com/science/article/pii/ S0032386103007961

A. Reuvers, J. van den Berg, C. Smolders, Formation of membranes by means of immersion precipitation: Part i. a model to describe mass transfer during immersion precipitation, Journal of Membrane Science 34 (1) (1987) 45 - 65. doi:https://doi.org/10.1016/S0376-7388(00)80020-4. 
URL http://www.sciencedirect.com/science/article/pii/ S0376738800800204

[10] Y. D. Kim, J. Y. Kim, H. K. Lee, S. C. Kim, Formation of polyurethane membranes by immersion precipitation. ii. morphology formation, Journal of Applied Polymer Science 74 (9) (1999) 2124-2132. doi:10.1002/ (SICI) 1097-4628(19991128) 74:9<2124: :AID-APP2>3.0.CO;2-Y. URL http://dx.doi.org/10.1002/(SICI)1097-4628(19991128)74: 9<2124: :AID-APP2>3.0.CO;2-Y

[11] G. A. R. Shojaie Saeed S., K. W. B., Dense polymer film and membrane formation via the dry-cast process part i. model development, Journal of Membrane Science 94 (1) (1994) 255 - 280. doi:https://doi.org/ 10.1016/0376-7388(93)E0228-C.

[12] B. Ladewig, Fundamentals of Membrane Processes, Springer Singapore, Singapore, 2017, pp. 13-37. doi:10.1007/978-981-10-2014-8_2. URL https : //doi .org/10.1007/978-981-10-2014-8_2

[13] J. W. Cahn, J. E. Hilliard, Free energy of a nonuniform system. i. interfacial free energy, The Journal of Chemical Physics 28 (2) (1958) 258267. arXiv:http://dx.doi.org/10.1063/1.1744102, doi:10.1063/ 1.1744102 .

URL http://dx.doi.org/10.1063/1.1744102

[14] P. Flory, Principles of Polymer Chemistry, Cornell university Press, 1953. 
[15] H. Manzanarez, J. Mericq, P. Guenoun, J. Chikina, D. Bouyer, Modeling phase inversion using cahn-hilliard equations -influence of the mobility on the pattern formation dynamics, Chemical Engineering Science (2017). doi:http://dx.doi.org/10.1016/j.ces.2017.08.009. URL http://wWW.sciencedirect.com/science/article/pii/ S0009250917305110

[16] B. Barton, P. Graham, J. McHugh, Dynamics of spinodal decomposition in polymer solutions near a glass transition, Macromolecules 31 (5) (1998) 1672 - 1679. doi:10.1021/ma970964j.

URL http://dx.doi .org/10.1021/ma970964j

[17] Y. Mino, T. Ishigami, Y. Kagawa, H. Matsuyama, Three-dimensional phase-phield simulations of membrane porous structure formation by thermally induced phase separation in polymer solutions, Journal of Membrane Science 483 (2015) 104 -111. doi:10.1016/j.memsci. 2015. 02.005 .

URL http://dx.doi.org/10.1016/j.memsci.2015.02.005

[18] B. Zhou, A. C. Powell, Phase field simulations of early stage structure formation during immersion precipitation of polymeric membranes in 2d and 3d, Journal of Membrane Science 268 (2) (2006) 150 - 164. doi:http://dx.doi.org/10.1016/j.memsci.2005.05.030. URL http://wwW.sciencedirect.com/science/article/pii/ S037673880500459X

[19] D. Tree, K. T. Delaney, H. D. Ceniceros, T. Iwama, G. Fredrickson, A 
multi-fluid model for microstructure formation in polymer membranes, Soft Matter 13 (2017) 3013-3030.

[20] D. Bouyer, O. M'Barki, C. Pochat-Bohatier, C. Faur, E. Petit, P. Guenoun, Modeling the membrane formation of novel pva membranes for predicting the composition path and their final morphology, AIChE Journal (2017) n/a-n/adoi:10.1002/aic.15670.

URL http://dx.doi.org/10.1002/aic.15670

[21] B. Barton, A. McHugh, Modeling the dynamics of membrane structure formation in quenched polymer solutions, Journal of Membrane Science 166 (1) (2000) 119 - 125. doi:https: //doi.org/10.1016/S0376-7388(99)00257-4. URL http://wwW.sciencedirect.com/science/article/pii/ S0376738899002574

[22] K.-W. D. Lee, P. K. Chan, X. Feng, Morphology development and characterization of the phase-separated structure resulting from the thermal-induced phase separation phenomenon in polymer solutions under a temperature gradient, Chemical Engineering Science 59 (7) (2004) 1491 - 1504. doi:https://doi.org/10.1016/j.ces.2003.12.025.

URL http://wwW.sciencedirect.com/science/article/pii/ S0009250904000594

[23] P. K. Chan, Effect of concentration gradient on the thermal-induced phase separation phenomenon in polymer solutions, Modelling Simul. Mater. Sci. Eng. 14 (1) (2006) 41-51. doi:10.1088/0965-0393/14/1/ 004 . 
634 [24] Y.-D. He, Y.-H. Tang, X.-L. Wang, Dissipative particle dynam635 ics simulation on the membrane formation of polymer-diluent system via thermally induced phase separation, Journal of Membrane Science 368 (1) (2011) 78 - 85. doi:https: //doi.org/10.1016/j.memsci.2010.11.010. URL http://wWW.sciencedirect.com/science/article/pii/ S037673881000863X

[25] Y. hui Tang, Y. dong He, X. lin Wang, Three-dimensional analysis of membrane formation via thermally induced phase separation by dissipative particle dynamics simulation, Journal of Membrane Science 437 (2013) 40 - 48 doi:https: //doi.org/10.1016/j.memsci.2013.02.018. URL http://wwW.sciencedirect.com/science/article/pii/ S0376738813001373

[26] Y. hui Tang, Y. dong He, X. lin Wang, Investigation on the membrane formation process of polymer-diluent system via thermally induced phase separation accompanied with mass transfer across the interface: Dissipative particle dynamics simulation and its experimental verification, Journal of Membrane Science 474 (2015) 196 - 206. doi:https://doi.org/10.1016/j.memsci.2014.09.034.

URL http://www.sciencedirect.com/science/article/pii/ S0376738814007364

[27] Y. hui Tang, H. han Lin, T. yin Liu, H. Matsuyama, X. lin Wang, Multiscale simulation on the membrane formation pro- 


\begin{tabular}{|c|c|c|}
\hline 658 & 0 & cess via thermally induced phase separation accompanied with \\
\hline 659 & & heat transfer, Journal of Membrane Science 515 (2016) $258-267$. \\
\hline 660 & & doi:https://doi.org/10.1016/j.memsci.2016.04.024. \\
\hline 661 & 口 & http://www.sciencedirect.com/science/article/pii/ \\
\hline 662 & & S0376738816302344 \\
\hline 663 & {$[28]$} & Y. hui Tang, E. Ledieu, M. R. Cervellere, P. C. Millett, D. M. Ford, \\
\hline 664 & 口 & X. Qian, Formation of polyethersulfone membranes via nonsolvent \\
\hline 665 & 口 & induced phase separation process from dissipative particle dynam- \\
\hline 666 & & ics simulations, Journal of Membrane Science 599 (2020) 117826. \\
\hline 667 & & doi:https://doi.org/10.1016/j.memsci.2020.117826. \\
\hline 668 & 口 & http://www.sciencedirect.com/science/article/pii/ \\
\hline
\end{tabular}

670 [29] A. Hanafia, C. Faur, A. Deratani, P. Guenoun, H. Garate, D. Quemener, 671 C. Pochat-Bohatier, D. Bouyer, Fabrication of novel porous 672 membrane from biobased water-soluble polymer (hydroxypropy673 lcellulose), Journal of Membrane Science 526 (2017) 212 - 220. 674 doi:https://doi.org/10.1016/j.memsci.2016.12.037.

675 URL http://wWw.sciencedirect.com/science/article/pii/ $676 \quad$ S0376738816316246

${ }_{677}^{6}[30]$ D. R. Tree, L. F. Dos Santos, C. B. Wilson, T. R. Scott, J. U. Garcia, 678 G. H. Fredrickson, Mass-transfer driven spinodal decomposition in a 679 ternary polymer solution, Soft Matter 15 (2019) 4614-4628. doi:10. $680 \quad 1039 /$ C9SM00355J.

681 URL http://dx.doi.org/10.1039/C9SM00355J 
[31] P. Debye, Angular dissymmetry of the critical opalescence in liquid mixtures, The Journal of Chemical Physics 31 (3) (1959) 680-687. arXiv: http://dx.doi.org/10.1063/1.1730446, doi:10.1063/1.1730446.

URL http://dx.doi.org/10.1063/1.1730446

[32] E. J. Kramer, P. Green, C. J. Palmstrøm, Interdiffusion and marker movements in concentrated polymer-polymer diffusion couples, Polymer 25 (4) (1984) 473 - 480. doi:10.1016/0032-3861(84)90205-2.

URL http://dx.doi .org/10.1016/0032-3861(84)90205-2

[33] H. Sillescu, Relation of interdiffusion and self-diffusion in polymer mixtures, Makromol. Chem. Rapid Commun 5 (1984) 519 - 523. doi: $10.1002 / \operatorname{marc} .1984 .030050906$.

URL http://dx.doi.org/10.1002/marc.1984.030050906

[34] de Gennes, Solvent evaporation of spin cast films: "crust" effects, P. Eur. Phys. J. E 7 (1) (2002) 31 - 34. doi:https://doi.org/10.1140/ epje/i200101169.

[35] M. Dušková-Smrčková, K. Dušek, P. Vlasák, Solvent activity changes and phase separation during crosslinking of coating films, Macromolecular Symposia 198 (1) (2003) 259-270. doi:10.1002/masy.200350822. URL http://dx.doi.org/10.1002/masy.200350822

[36] G. Ovejero, M. D. Romero, E. Díez, I. Díaz, P. Pérez, Thermodynamic modeling and simulation of styrene-butadiene rubbers (sbr) solvent equilibrium staged processes, Industrial \& Engineering Chemistry Research 48 (16) (2009) 7713-7723. arXiv:http://dx.doi.org/10. 
1021/ie9006497, doi:10.1021/ie9006497.

URL http://dx.doi.org/10.1021/ie9006497

[37] D. Bouyer, C. Pochat-Bohatier, Validation of mass-transfer model for vips process using in situ measurements performed by near-infrared spectroscopy, AIChE Journal 59 (2) (2013) 671-686. doi:10.1002/aic. 13839 .

URL http://dx.doi.org/10.1002/aic.13839

[38] C. Tsay, A. McHugh, Mass transfer dynamics of the evaporation step in membrane formation by phase inversion, Journal of Membrane Science 64 (1) (1991) 81 - 92. doi:https: //doi.org/10.1016/0376-7388(91)80079-L. URL http://wwW.sciencedirect.com/science/article/pii/ $037673889180079 \mathrm{~L}$

[39] K. Ozawa, T. Okuzono, M. Doi, Diffusion process during drying to cause the skin formation in polymer solutions, Japanese Journal of Applied Physics 45 (11) (2006) 8817-8822. doi:10.1143/jjap.45.8817.

URL https://doi .org/10.1143\%2Fjjap.45.8817

[40] R. Rabani, M. H., P. Dauby, A phase-field model for the evaporation of thin film mixtures, Phys. Chem. Chem. Phys. 22 (12) (2017) 6638 6652. doi:10.1039/D0CP00214C.

URL http://dx.doi.org/10.1039/D0CP00214C

[41] J. Cummings, J. Lowengrub, B. Sumpter, S. Wise, R. Kumar, Modeling solvent evaporation during thin film formation in phase separat- 
728 ing polymer mixtures, Soft Matter 45 (11) (2018) 8817-8822. doi:

$729 \quad 10.1039 / \mathrm{c} 7 \mathrm{sm} 02560 \mathrm{~b}$.

730 URL https://doi.org/10.1039/c7sm02560b

731 [42] P. Guenoun, D. Beysens, M. Robert, Dynamics of wetting and phase 732 separation, Phys. Rev. Lett. 65 (1990) 2406-2409. doi:10.1103/

${ }_{733}$ PhysRevLett.65.2406.

${ }_{734}$ URL https://link.aps.org/doi/10.1103/PhysRevLett.65.2406

${ }_{735}^{6}$ [43] R. C. Ball, R. L. H. Essery, Spinodal decomposition and pattern forma-

736 tion near surfaces, Journal of Physics: Condensed Matter 2 (51) (1990)

${ }_{737}$ 10303-10320. doi:10.1088/0953-8984/2/51/006.

738

URL https://doi.org/10.1088\%2F0953-8984\%2F2\%2F51\%2F006 